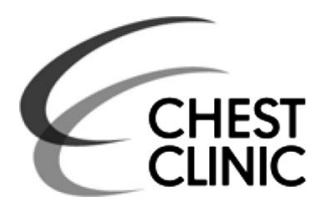

published online only. To view please visit the journal online (http://dx.doi.org/10.1136/ thoraxjnl-2015-207352).

${ }^{1}$ Department of Radiology, Barnet General Hospital, Royal Free London NHS Foundation

Trust, London, UK

${ }^{2}$ Department of Respiratory Medicine, Barnet General Hospital, Royal Free London NHS Foundation Trust, London, UK

\section{Correspondence to} Dr S S Hare, Department of Radiology, Barnet Hospital, Royal Free London NHS

Foundation Trust,

Wellhouse Lane, EN5 3DJ, UK; samhare@nhs.net

Received 28 May 2015 Revised 16 June 2015 Accepted 24 June 2015 Published Online First 14 July 2015

\title{
Ambulatory percutaneous lung biopsy with early discharge and Heimlich valve management of iatrogenic pneumothorax: more for less
}

\author{
A N Tavare, ${ }^{1}$ D D Creer, ${ }^{2}$ S Khan, ${ }^{2}$ R Vancheeswaran, ${ }^{2}$ S S Hare ${ }^{1}$
}

\section{ABSTRACT}

A prospective study of 489 consecutive outpatient imageguided percutaneous lung biopsies was conducted to determine whether early discharge, incorporating ambulatory Heimlich valve drain, is potentially advantageous to the National Health Service. Patients were discharged at 30 or $60 \mathrm{~min}$, with significant pneumothoraces treated using Heimlich valve. 485 $(99.2 \%)$ patients were successfully discharged early, 402 at 30 min. 87 (17.8\%) patients developed pneumothorax: 52 required Heimlich valve; 5 proceeded to biopsy with Heimlich valve in situ. All drains were removed within $48 \mathrm{~h}$ $38 / 52(73.1 \%)$ at $24 \mathrm{~h}$. Our results provide evidence for a paradigm shift in UK practice: early discharge lung biopsy, facilitated by ambulatory Heimlich valve, is safe with significant clinical and economic benefits.

\section{INTRODUCTION}

Image-guided percutaneous lung biopsy (PLB) is pivotal in the diagnosis of lung lesions. There is dramatically escalating clinical demand for tissue diagnosis, even in comorbid patients, partly due to the emergence of non-surgical lung cancer treatments but also prior to surgical resection. Unfortunately, UK practice has failed to evolve in tandem with these treatment advances. ${ }^{1}$

Pneumothorax is common post PLB, particularly as many patients have coexisting emphysema. There is little consensus regarding post-biopsy chest X-ray (CXR) timing to exclude significant pneumothorax and thereby ensure safe discharge. UK practice is highly variable ${ }^{2}$ with protocols that routinely require several hours (typically 3-6) of bed rest and a 4 h post-biopsy CXR.

A 2001 Canadian study ${ }^{3}$ outlining successful use of early discharge, with Heimlich valve chest drain (HVCD) management of iatrogenic pneumothorax, failed to impact UK PLB practice. ${ }^{4}$ Consequently, the 62-day lung cancer treatment initiation target remains under severe pressure due to hospital bed requirement for prolonged post-biopsy monitoring under usual UK practice. ${ }^{5}$

The purpose of this study was to determine whether a radiology-led early discharge PLB service, incorporating ambulatory outpatient small calibre HVCD to treat pneumothorax, is potentially safe and advantageous to the National Health Service (NHS).

\section{METHODS}

Between March 2011 and March 2015, we conducted a prospective evaluation of 489 consecutive outpatient PLBs, all performed using an HVCDfacilitated early discharge outpatient protocol (see online supplementary figure S1). Data on complications (pneumothorax rates; HVCD rates; failed early discharge) were collected.

Patients were scheduled into $30 \mathrm{~min}$ slots. No hospital beds were pre-emptively booked. Biopsies were performed under either fluoroscopy or CT guidance: masses $>3 \mathrm{~cm}$, nodules $>1 \mathrm{~cm}$ visible on CXR and Pancoast tumours tended to be biopsied fluoroscopically. The only absolute biopsy contraindication was anticoagulation therapy (international normalised ratio $<1.5$ and platelets $>50 \times 10^{9} / \mathrm{L}$ deemed suitable to proceed). Patients were instructed to stop antiplatelet agents 5 days pre-procedure if feasible. Pulmonary function tests (PFTs) were not routinely performed preprocedure, and there was no minimum $\mathrm{FEV}_{1}$ limitation. ${ }^{3}$

Co-axial technique PLB was performed using $10 \mathrm{~mL} 1 \%$ lignocaine; 19G co-axial introducer needle (Argon Medical, Texas, USA); 20G core biopsy needle (Pro-mag (Argon Medical)) connected to Pro-mag biopsy gun (Angiotech, Florida, USA). At least three core biopsies were obtained for each lesion.

A post-biopsy posteroanterior (PA) erect $30 \mathrm{~min}$ CXR was obtained in all patients: (a) asymptomatic patients without significant pneumothorax were discharged at $30 \mathrm{~min}$ with an information leaflet (see online supplementary appendix); (b) patients with small asymptomatic pneumothorax were instructed to walk around the department with repeat CXR at $60 \mathrm{~min}$. If the pneumothorax was smaller/stable, and asymptomatic, early discharge was initiated as above; (c) in cases of large/symptomatic 30 min pneumothorax; enlarging pneumothorax at $60 \mathrm{~min}$; or persisting, symptomatic pneumothorax, an $8 \mathrm{Fr}$ drain (Angiotech, Florida, USA) was inserted by the radiologist into the second intercostal space, mid-clavicular line using local anaesthesia and trochar technique. The external end of the drain was connected to a Heimlich valve (HV) (Vygon, Ecouen, France) (figure 1). A bubble test (end of HV placed into a cup of water and patient instructed to cough) was performed and explained to the patient, with a positive bubble test confirming satisfactory drain position. No repeat CXR was performed. All management took place entirely within the radiology department without formal respiratory physician input. 
Figure 1 (A) A number of Heimlich valves are commercially available (left to right: Becton, Dickinson and Company (New Jersey, USA), Vygon (Ecouen, France) and Cook Medical (Dublin, Ireland)) ranging in size from 11 to $23 \mathrm{~cm}$ in length. (B) An in situ $8 \mathrm{~F}$ chest drain connected to a Heimlich valve chest drain (HVCD) is shown in a patient who underwent dual-site CT-guided biopsy, all at the same sitting, of a $17 \mathrm{~mm}$ right upper lobe nodule and a right-sided basal pleural nodule. HVCD was inserted post-percutaneous lung biopsy due to a symptomatic right pneumothorax. The drain was removed at $24 \mathrm{~h}$ after a negative bubble test. Biopsy revealed metastatic pulmonary adenocarcinoma.
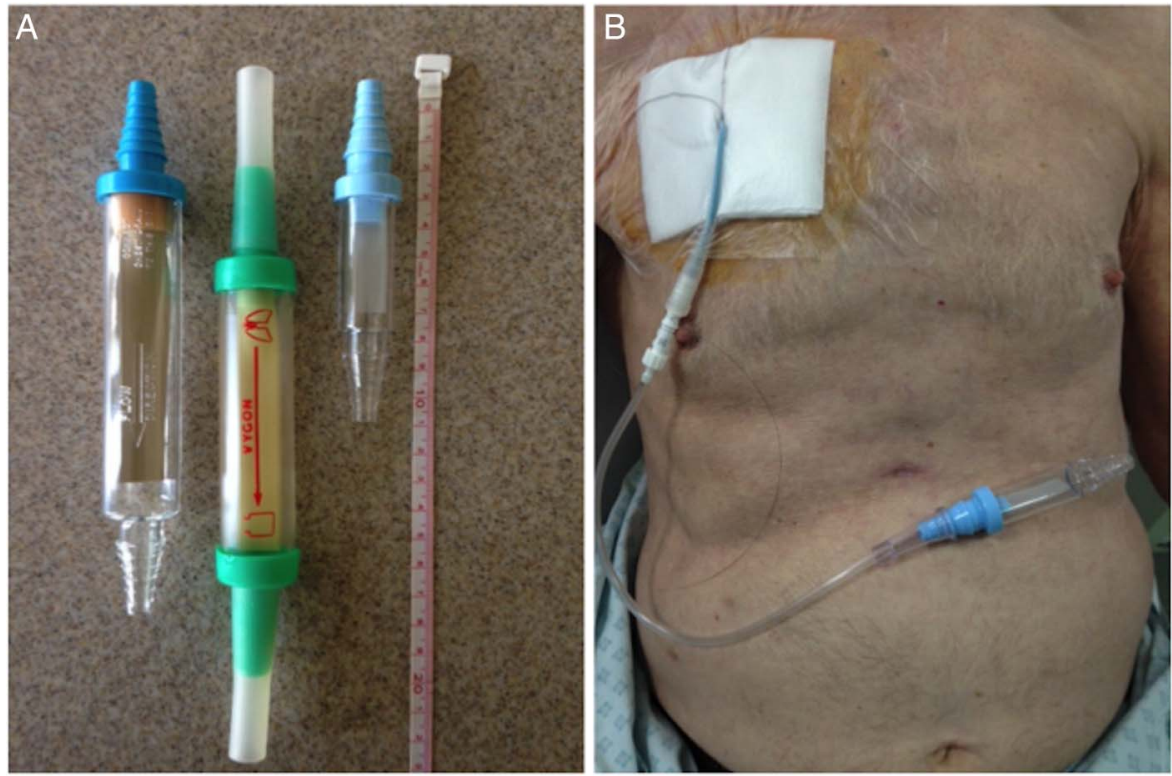

Patients were discharged with HVCD in situ with instructions to perform a home $24 \mathrm{~h}$ bubble test and return to the radiology department for drain removal if negative (ie, pneumothorax resolution). In the event of an ongoing positive $24 \mathrm{~h}$ bubble test, patients were asked to perform further bubble tests each subsequent morning and return for drain removal once negative. After drain removal, no repeat CXR was performed and patients were discharged with verbal advice. No patients re-presented to hospital with recurrent pneumothorax post drain removal.

\section{RESULTS}

In total, 489 PLBs were performed: 254 under CT guidance and 235 under fluoroscopy guidance (table 1).

In total, 402 patients $(82.2 \%)$ were successfully discharged at $30 \mathrm{~min}$. Our four unplanned admissions, all discharged the following morning, were elderly patients living alone, treated with HVCD, but preferring overnight hospital admission.

Thirty-five patients, successfully discharged at $60 \mathrm{~min}$ with stable, asymptomatic pneumothorax, were contacted by telephone $24 \mathrm{~h}$ later and reported no subsequent complications. Fifty-two

Table 1 Results and complications of 489 consecutive outpatient biopsies performed during study period

\begin{tabular}{|c|c|}
\hline & Number (\% of total biopsies) \\
\hline \multicolumn{2}{|l|}{ Results } \\
\hline Successful early discharge & $485(99.1 \%)$ \\
\hline Early 30 min discharge & $\begin{array}{l}402(82.2 \%) \\
\text { (all without further incident) }\end{array}$ \\
\hline Diagnostic histology result & $478(97.8 \%)$ \\
\hline \multicolumn{2}{|l|}{ Complications } \\
\hline Pneumothorax & $87(17.8 \%)$ \\
\hline Pneumothorax — suitable for $60 \mathrm{~min}$ discharge without requiring intervention & $\begin{array}{l}35(7.2 \%) \\
\text { (all without further incident) }\end{array}$ \\
\hline Pneumothorax - treated with HVCD insertion & $\begin{array}{l}52(10.6 \%) \\
(5 / 52 \text { intra-biopsy; } 36 / 52 \text { at } 30 \mathrm{~min} ; 11 / 52 \text { at } 60 \text { min after mild exertion) }\end{array}$ \\
\hline HVCD drain removal (negative bubble test) & $\begin{array}{l}38 / 52 \text { at } 24 \mathrm{~h} ; 14 / 52 \text { at } 48 \mathrm{~h} \\
0 / 52>48 \mathrm{~h}\end{array}$ \\
\hline HVCD requiring intra-pleural bupivacaine for chest tube pain management & $\begin{array}{l}8 \\
\text { (overall } 1.6 \% ; 15.4 \% \text { of those treated with HVCD) }\end{array}$ \\
\hline Pneumothorax—requiring hospital admission & 0 \\
\hline Small volume haemoptysis $<30$ min managed conservatively & $17(3.8 \%)$ \\
\hline Small volume haemoptysis $>30$ min post biopsy & 0 \\
\hline Massive haemoptysis & 0 \\
\hline Systemic arterial air embolism & 0 \\
\hline Mortality & 0 \\
\hline Biopsy performed with HVCD in situ & $\begin{array}{l}5(1.0 \%) \\
\text { ( } 5 / 5 \text { drains removed after negative bubble test at } 24 \mathrm{~h} \text { post biopsy) }\end{array}$ \\
\hline Requiring hospital admission post HVCD insertion & $4(0.8 \%)$ (for social issues) \\
\hline Re-presenting to hospital with pneumothorax after HVCD removal & 0 \\
\hline
\end{tabular}


patients were treated with HVCD insertion, with $47 / 52$ performed post procedure. Also, 5/52 patients (slim with significant emphysema) developed symptomatic pneumothorax, necessitating rapid HVCD insertion, during local anaesthesia itself: three of these were immediately biopsied with HVCD in situ. The other two patients were discharged home and returned the following day for uneventful, successful PLB with HVCD in situ.

Eight patients developed HVCD-related pleuritic pain and were successfully managed with $10 \mathrm{~mL}$ of intrapleural $0.5 \%$ bupivacaine. CXRs revealed swift, near complete, re-expansion of the lung post-HVCD insertion. We postulate that these 'pleurisy' symptoms related to drain and pleural contact.

The radiologist was blinded to formal PFTs at time of biopsy. A retrospective review of PFT data at the end of the study period, available in $212 / 489$ patients, revealed 28 with $\mathrm{FEV}<1 \mathrm{~L}$ (range $0.39-0.99)$. Also, 22/28 (78.6\%) were discharged at $30 \mathrm{~min}$ without incident; $6 / 28$ patients $(21.4 \%)$ developed post-PLB pneumothorax with $3(10.7 \%)$ requiring outpatient HVCD, for 24 h duration.

\section{DISCUSSION}

Our results demonstrate that a radiology-managed early discharge PLB service, using HVCD for significant pneumothorax, is safe and effective even in patients with severe emphysema. Although such patients may not be surgical candidates, histology is desirable because of the emergence of non-surgical treatments such as radiofrequency ablation (RFA), stereotactic ablative radiotherapy and gene-targeted drug therapies.

Central to our protocol is HVCD management of pneumothorax. This device is small enough (figure 1) to be discreetly placed under the patient's clothes, allowing normal daily activities. Our research indicates these are not currently used in the UK for post-biopsy pneumothorax. Our threshold for HVCD insertion is lower if a transfissural biopsy track is used (ie, four pleural surfaces crossed). We advocate an aggressive approach to drainage, preferring to insert a low morbidity ambulatory HVCD, which allows rapid and safe discharge, rather than risk a delayed significant pneumothorax at home, particularly in comorbid patients. As such we make patients with small pneumothoraces on $30 \mathrm{~min}$ CXR mildly exert themselves prior to a 60 min CXR to gauge 'stability' of the pneumothorax, thereby guiding the decision to insert HVCD prior to discharge.

Incorporating use of HVCD not only allows effective outpatient management of significant pneumothorax, but crucially enables biopsy of more difficult lesions with no defined lower limit of $\mathrm{FEV}_{1}$. Notably the complexity of referrals has increased with our practice (eg, smaller, deeper and subsolid lesions). Our overall rate of pneumothorax $(17.8 \%$, with $10.6 \%$ requiring drainage) falls within published rates (17-26.6\% and 1-14.2\%, respectively). ${ }^{1}$ Moreover, many UK departments still decline PLB in patients with severe emphysema due to the risk of compromising pneumothorax in low respiratory reserve. In our method, however, the availability of HVCD means such patients are never declined PLB as even significant pneumothorax can be swiftly and effectively managed in an early discharge outpatient fashion. Consequently, insistence on formal pre-procedure $\mathrm{FEV}_{1}$ should not delay biopsy. In fact, our patients are routinely consented prebiopsy for continuing PLB with HVCD in situ in the event of an immediate significant pre-biopsy or intra-biopsy pneumothorax (see online supplementary figure S2). To our knowledge, this approach is also a first for UK and European PLB practice.

Access to PLB under standard UK practice is dependent on hospital bed availability. Our service eliminates bed-related scheduling delays and therefore facilitates swifter lung cancer diagnosis. Furthermore, preoperative histology from PLBs in our study has frequently obviated the need for intraoperative frozen section, thereby saving an average of 40 min per operation in addition to reducing the potential for expensive litigation.

Our practice was established by a single thoracic radiologist but is being strengthened through training of two further interventional radiologists. There is a marginally higher time commitment for the radiologist (15 min extra per case due to post-PLB CXR review and potential HVCD insertion). However, compared with conventional practice, this early discharge protocol requires a maximum of $60 \mathrm{~min}$ ambulatory hospital stay, entirely within the imaging department, corresponding to a cumulative saving of between 550 and 750 bed hours/year in uncomplicated lung biopsy. In addition, a successful early discharge rate of $>99 \%$ substantially reduces respiratory clinical service workload due to HVCD management of significant post-biopsy pneumothorax with immediate patient discharge. The service has further saved, at minimum, 52 respiratory inpatient bed days compared with standard UK practice (underwater seal chest drain management of post-biopsy pneumothorax). This figure is an underestimate as prolonged drainage is often required. The cost of ambulatory $\mathrm{HVCD}$ at $£ 36$ ( $£ 13 \mathrm{HV} ; £ 23$ drain), coupled with outpatient management, compares favourably to other UK departments performing lung biopsy (as well as to our own practice pre-2011) where management of post-PLB pneumothorax costs at least $£ 392$ ( $£ 92$ underwater seal chest drain kit plus $£ 300$ per hospital bed day).

Patient feedback regarding this service is strongly positive with patients commenting on how easy it is to perform the home bubble test.

\section{CONCLUSION}

This novel approach to PLB in the NHS setting provides an elegant solution to managing patients: early discharge is safe and expeditious; allows greater volumes of biopsy per institution; and outpatient HVCD saves precious hospital beds while enabling biopsy in severely emphysematous patients with negligible morbidity.

These results can be extrapolated to the NHS, in both small and large hospital settings. Scaling this approach more widely would help provide earlier lung cancer diagnosis with considerably increased patient throughput. Given the parlous financial state of the NHS, it is significant that these operational efficiencies can be achieved with cost and resource savings while yielding significant advantages for patients, respiratory services and healthcare systems.

Contributors SSH performed the biopsies. ANT and SSH wrote the manuscript. DDC, SK and RV were the clinicians responsible for patient referral and helped write the manuscript.

Competing interests None declared.

\section{Patient consent Obtained.}

Provenance and peer review Not commissioned; internally peer reviewed.

\section{REFERENCES}

1 Manhire A, Charig M, Clelland C, et al. Guidelines for radiologically guided lung biopsy. Thorax 2003;58:920-36.

2 Richardson CM, Pointon KS, Manhire AR, et al. Percutaneous lung biopsies: a survey of UK practice based on 5444 biopsies. Br J Radiol 2002;75:731-5.

3 Dennie CJ, Matzinger FR, Marriner JR, et al. Transthoracic needle biopsy of the lung: results of early discharge in 506 outpatients. Radiology 2001;219:247-51.

4 Brims FJ, Maskell NA. Ambulatory treatment in the management of pneumothorax: a systematic review of the literature. Thorax 2013;68:664-9.

5 NHS England. Provider-based Cancer Waiting Times for Q3 2014-15. http://www. england.nhs.uk/statistics/2015/02/18/

provider-based-cancer-waiting-times-for-q3-2014-15/ (accessed 4 Feb 2015). 